\title{
3D ANALYSIS OF LOW-VOLTAGE GAS-FILLED DC SWITCH USING SIMPLIFIED ARC MODEL
}

\author{
S. Gortschakow ${ }^{a, *}$, D. Gonzalez ${ }^{a}$, S. Yu ${ }^{b}$, F. Werner ${ }^{b}$ \\ a Leibniz institute for plasma science and technology (INP), Felix-Hausdorff-Strasse 2, 17489 Greifswald, \\ Germany \\ b TDK Electronics, Gradestrasse 48-50, 12347 Berlin, Germany \\ * sergey.gortschakow@inp-greifswald.de
}

\begin{abstract}
Electro-magnetic simulations have been used for the visualization of distribution of Lorentz force acting on a DC switching arc in low-voltage contactor. A simplified plasma model (black-box model) was applied for the description of arc conductivity. Arc geometry was gained from the high-speed camera images. Influence of arc position, arc current and of external magnetic field has been studied. Results have been compared with optical observations of the arc dynamics.
\end{abstract}

Keywords: black-box model, Lorentz force, arc dynamics, 3D simulations.

\section{Introduction}

3D numerical simulations have a great potential for optimization of switching devices. The main drawback of such simulations is the complexity of the arc model, which must be applied in order to get the realistic results. Consideration of arc dynamics, changes in plasma composition, high temperature and pressure gradients requires enormous mathematical efforts and immense computing power. However, some questions of the design optimization, such as positioning of additional elements for arc control, influence of the electrode shape on the resulting distribution of electromagnetic fields can be roughly solved applying suitable black-box arc models.

Increasing use of pure electric as well as of hybrid cars promotes the research on DC switching devices and contactors with enhanced current interruption capability. Recent studies of DC switching devices in the gas mixtures [1], have shown that hydrogen mixtures, especially those with nitrogen exhibit a higher electric strength as well as a shorter arcing time comparing to air. Additional application of magnetic arc blowing causes further reduction of arcing time. According to the results of [1], the mixture with proportion of $80 \% \mathrm{H}_{2}$ and $20 \% \mathrm{~N}_{2}$ is the most promising for DC applications. In other publications, like e.g. [2] the influence of working pressure and comparison between several gases $\left(\mathrm{H}_{2}, \mathrm{~N}_{2}\right.$, He and air) has been discussed putting again the mixture $\mathrm{H}_{2} / \mathrm{N}_{2}$ as a favourite due to significantly faster arc interruption (up to 7 time comparing to $\mathrm{He}$ ). The arc duration was inversely proportional to working pressure. In $[3,4]$ the advantages of magnetic field blowing for DC air circuit breakers and relays for operating voltages below $1000 \mathrm{~V}$.

Present contribution focusses on numerical simulations of DC switching arc in low-voltage contactor. Electro-magnetic simulations have been used for the visualization of distribution of Lorentz force in order to understand the general arc dynamics. Arc geometry was gained from the high-speed camera images. The influence of external magnets and of the current density was studied using a simple arc model. Qualitative comparison with corresponding experiments has been performed for model validation. The results are in good agreement and confirm the ability of used method.

\section{Arc diagnostics}

Numerical analysis has been performed for a model arc chamber presented schematically in Figure 1. The electrode system consists of double bridge contactor with two fixed contacts and moving bridge made of copper. Maximum electrode distance is set between and $3 \mathrm{~mm}$. The chamber is made of plexiglass in order to get an optical access to the arc keeping all mechanical parts from original DC contactor. The chamber is filled with mixture $\mathrm{H}_{2} / \mathrm{N}_{2}$ in $80 \% / 20 \%$ proportion to a total pressure between 1 and 7 bar.

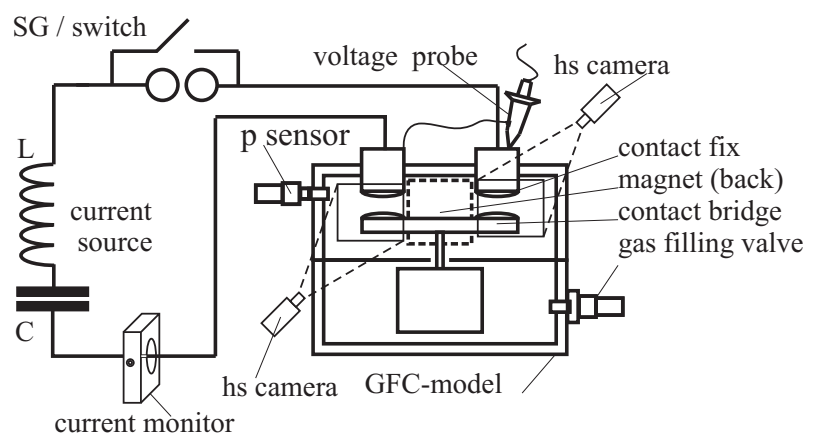

Figure 1. Schematic view of experimental setup and used diagnostics.

The diagnostics comprises electric measurements (Tektronix TCP202 Hall current probe, Tektronix $6015 \mathrm{~A}$ voltage probe), pressure measurements (piezoelectric sensor PCB-105C), optical observation of arc 
dynamics by a high-speed camera (RedLake Motion Pro Y6) with frame rate of $4000 \mathrm{fps}$ and acquisition time of $1 \mu$ s as well as acquisition of spatially integrated arc spectra using compact spectrometer (Avantes VIS-spectrometer, AvaSpec-ULS2048).

Detailed results of experimental diagnostics are presented elsewhere [5]. In the present paper mainly the results of arc dynamics observations are utilized.
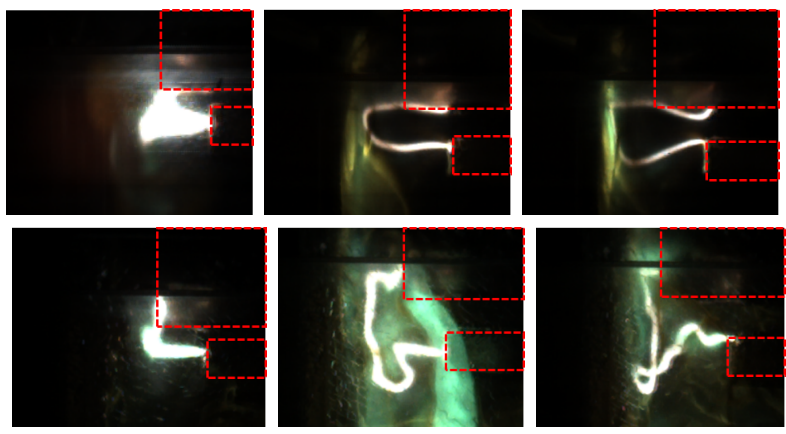

Figure 2. Arc images: upper row - without external magnets, lower row - with magnets. Red lines show schematically positions of bridge and left electrode.

Images of the switching arc for two variants without and with external magnets are shown in Fig. 2 for the current of $80 \mathrm{~A}$. The arc is elongated for both geometries and in addition twisted when the permanent magnets have been used. Notice, that according to results of [6] the influence of magnetic fields causes not only the arc elongation through Lorentz force but also an increase of thermal losses due to created turbulent cooling. This leads to the reduction of arc temperature and consequently of plasma conductivity and, hence to more effecting switching-off process. Another positive effect of the arc column twisting is that the arc is kept away from the wall. This leads to the reduction of the wall erosion.

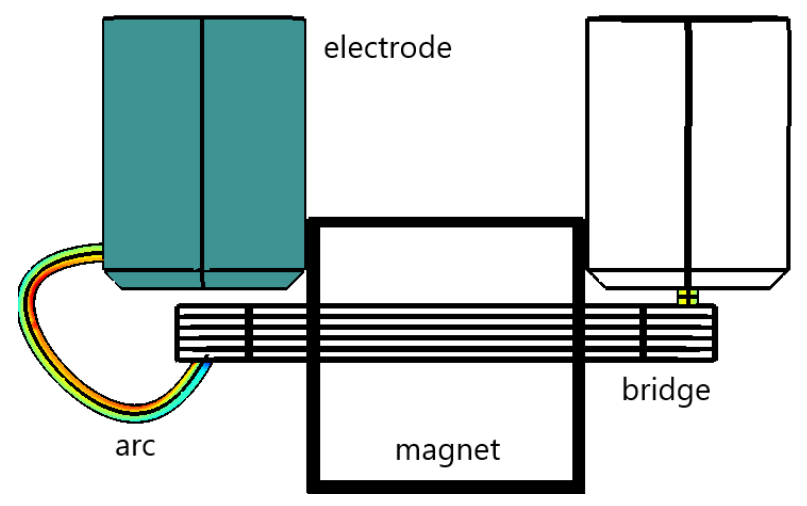

Figure 3. Model geometry of arc chamber.

\section{Description of the model}

Figure 3 presents the model geometry used in simulations. It consists from two cylindrical electrodes, bridge electrode, permanent magnets and arc plasma. The distance between the electrodes is fixed to $2 \mathrm{~mm}$.
The system of Maxwell equations has been used to model the electro-magnetic field distribution. Taking into account the assumptions for the arc plasma (corresponding conductivity model) the following equation system was used for the determination of the electric potential $V$, magnetic vector potential $\mathbf{A}$ and magnetic field strength $B$ :

$$
\begin{gathered}
\nabla \cdot(\sigma \nabla V)=0 \\
\nabla^{2} \mathbf{A}=\mu \sigma \nabla V \\
\mathbf{B}=\nabla \times \mathbf{A}+\mathbf{B}_{r}
\end{gathered}
$$

where $\sigma$ is the electric conductivity, $\mu$ is permeability of the medium and $\mathbf{B}_{r}$ denotes the magnetic flux provided by external magnetic elements. Finally, the electromagnetic force $\mathbf{F}_{\mathrm{L}}$ (Lorentz force) which is responsible for arc plasma movement is determined by following relation

$$
\mathbf{F}_{\mathrm{L}}=\mathbf{j} \times \mathbf{B}
$$

with the current density

$$
\mathbf{j}=-\sigma \nabla V
$$

The system was complemented by appropriate boundary conditions, i.e. ground potential at one electrode, fixed current at the second cylindrical electrode as well as zero current density and magnetic insulation at the outer boundaries. The model was developed using the commercial code COMSOL Multiphysics Version 5.4. The combination of modules „Electric currents" and „Magnetic fields" has been used to describe the DC arc.
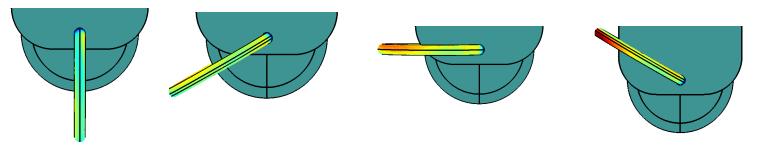

Figure 4. Chosen arc positions in the electrode system, rotation by 0, 60, 90 and 120 degree. Bottom view from bridge electrode.

\subsection{Arc plasma model}

A simplified plasma model (black-box model) has been used for the investigations. The shape of the arc was chosen based on experimental observations. The initial stage of arc development during the contact separation corresponds to an cylindrical and axial symmetric plasma. Then, due to the action of Lorentz force the arc elongation begins (cf. images in the first column in Fig. 2). This shape was chosen for numerical analysis. The arc column is assumed to has a form of sleeve with homogeneous conductivity distribution in axial and radial directions direction. Since the arc plasma shows high dynamics, several positions (Fig. 4 have been analysed.

The black-box model has several parameters: arc diameter, arc shape (length) and the conductivity. The arc length was fixed from the images of the arc, 
as mentioned above. The arc diameter was estimated also from images and assumed to be of about $1 \mathrm{~mm}$. As the experimental results [5] clarify, the arc spectrum includes intensive lines of copper. It is well known that the presence of only few percent admixture of metal vapour in the plasma conductivity becomes close to that of pure metal plasma. Furthermore, the temperature of metal affected plasma is in the range $8000-12000 \mathrm{~K}$. Taking into account those aspects the plasma conductivity was estimated to be around $10^{4} \mathrm{~S} / \mathrm{m}$.

\section{Results and discussion}

\subsection{Influence of external magnets}

Figure 5 shows the comparison of simulation results for the cases without and with external magnets. The colour scale gives the information about the magnitude of Lorentz force, while the arrows give the force direction. The length of the arcs is proportional to the magnitude of $F_{\mathrm{L}}$.

In the case without external magnets the arc always moves straight to the chamber wall. The same tendency was observed in the experiments (cf. Fig.2 upper row) where the arc reaches the wall after certain time. Such a behaviour finally leads to wall erosion and contamination of the walls with metal vapour. In term of optimization of switching behaviour, the walls should be made of gassing polymers in order to hold the arc away from the wall and to keep the surface clean. Application of external magnets leads to several effects in the arc dynamics. Firstly, the magnitude of Lorentz force increases by about factor of two for the positions 0 and 60 degree and by about factor of three for 90 and 120 degree which are close to the magnet. The second, more important impact, is the change of force distribution along the arc and of its direction. The sections of the arc column experience different direction and magnitude. This causes more efficient increase of the arc length and additional twisting of the column (cf. Fig. 2 lower row). Thus, the arc is kept away from the walls and has longer length providing more efficient arc cooling, shorter arcing time and, therefore, more effective current termination. The results for the arc positions 90 and 120 degree detect also the potential danger for these arc positions. Hot plasma is pushed toward the bridge electrode and, hence, can comes close to the central part of the device, which is not favour position for the arc burning. Fortunately, the experiments [5] have clarified, that such a scenario occurs quite seldom. Nevertheless, one has to keep this possibility in mind and to provide corresponding countermeasures.

\subsection{Influence of arc current}

Influence of current magnitude on the distribution of electromagnetic force is shown in Fig. 6. An increase of the arc current causes as expected to a proportional growth of the Lorentz force magnitude. Also, the distribution of force magnitude and direction along the arc length change. When the current increases, the self-induced magnetic field start to compete with external magnetic field. Finally, at very high currents the self-induced field becomes dominant and the spatial distribution and the direction of Lorentz force will be close to that presented in upper row of Fig. 5. The case with $400 \mathrm{~A}$ is still far away from this situation. However, the change of force direction is clearly visible. The results show the advantages of enhanced current in case of switching-off process: the arc will be more elongated and stronger twisted. In addition, the electromagnetic force for positions 90 and 120 degree is directed away from the bridge electrode.

\section{Summary}

The present contribution discusses the influence of additional arc control elements in model arc chamber. The shape of the arc plasma was adopted from experimental observations by high-speed camera. Distribution of electromagnetic fields and forces was calculated for various arc positions. It was found that external permanent magnets causes the twisting and elongation of the arc plasma and, therefore, positively influence the switching-off process. However, when the arc current increases the magnetic filed of the arc becomes stronger and the arc is less influenced by external magnets. This means that for higher current some additional arc extinguishing elements, like e.g. splitter plates or gassing polymers have to be used. The results of simulations have been compared with experimental observations of arc dynamics. A good qualitative agreement was found which gives the validation of applied approach.

\section{References}

[1] Y. Shiba et al. Study of DC Circuit breaker of $\mathrm{H}_{2}-\mathrm{N}_{2}$ Gas Mixture for High Voltage. Electrical Engineering in Japan, 174(2):1407-1413, 2011.

doi:10.1002/eej. 21042 .

[2] K. Sawa et al. Influence of sealed gas and its pressure on arc discharge in electromagnetic contactor. IEEE

Holm Conference on Electrical Contacts, pages 236-241, 2017. doi:10.1109/HOLM. 2017.8088093.

[3] Breaking performance of a circuit breaker influenced by a permanent magnetic field at dc voltages up to $450 \mathrm{v}$. Proceedings of the ICEC-ICREPE, pages 236-241, 2012.

[4] D. Volm and F. Winkler. Development of a compact relay for high voltage switching of up to $1000 \mathrm{v}$ and $40 \mathrm{a}$. International Conference on Electrical Contacts, 27:144-148, 2014.

[5] D. Gonzalez, S. Gortschakow, S. Yu, and F. Werner. Investigation of dc switching arc characteristics in hydrogen containing gas mixtures. acceptred for publication in Plasma Physics and Technology, 2019.

[6] J. Shea, E. Heckman, and J. Suarez Guevara. DC Arc Properties in a DC Magnetic Fields. 64th IEEE Holm Conference on Electrical Contacts/29th International Conference on Electrical Contacts, 64(1):195-202, 2017. 

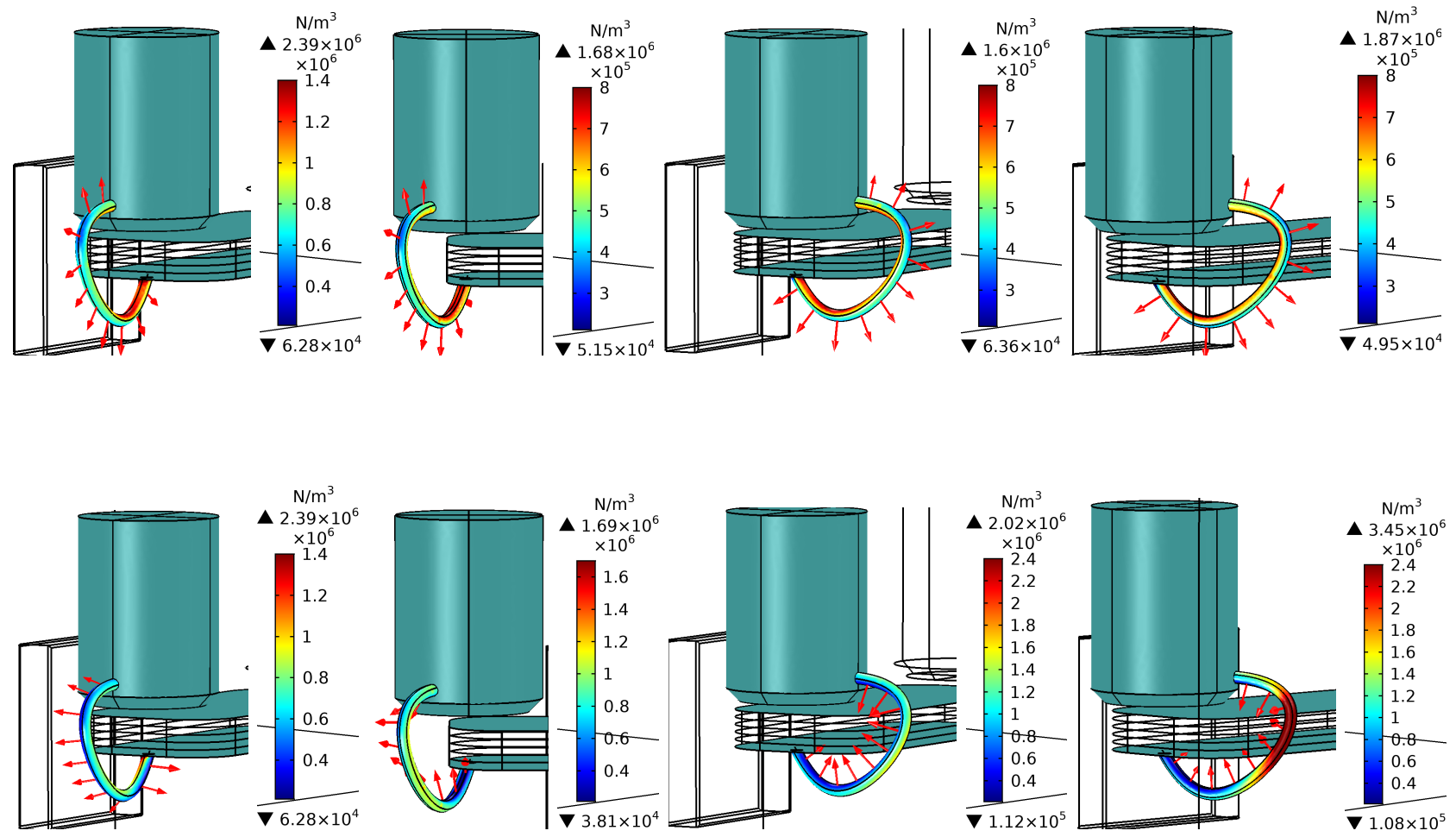

Figure 5. Lorentz force distribution in case without (upper row) and with (lower row) external magnets. Arc positions (from left to right) 0, 60, 90 and 120 degree.
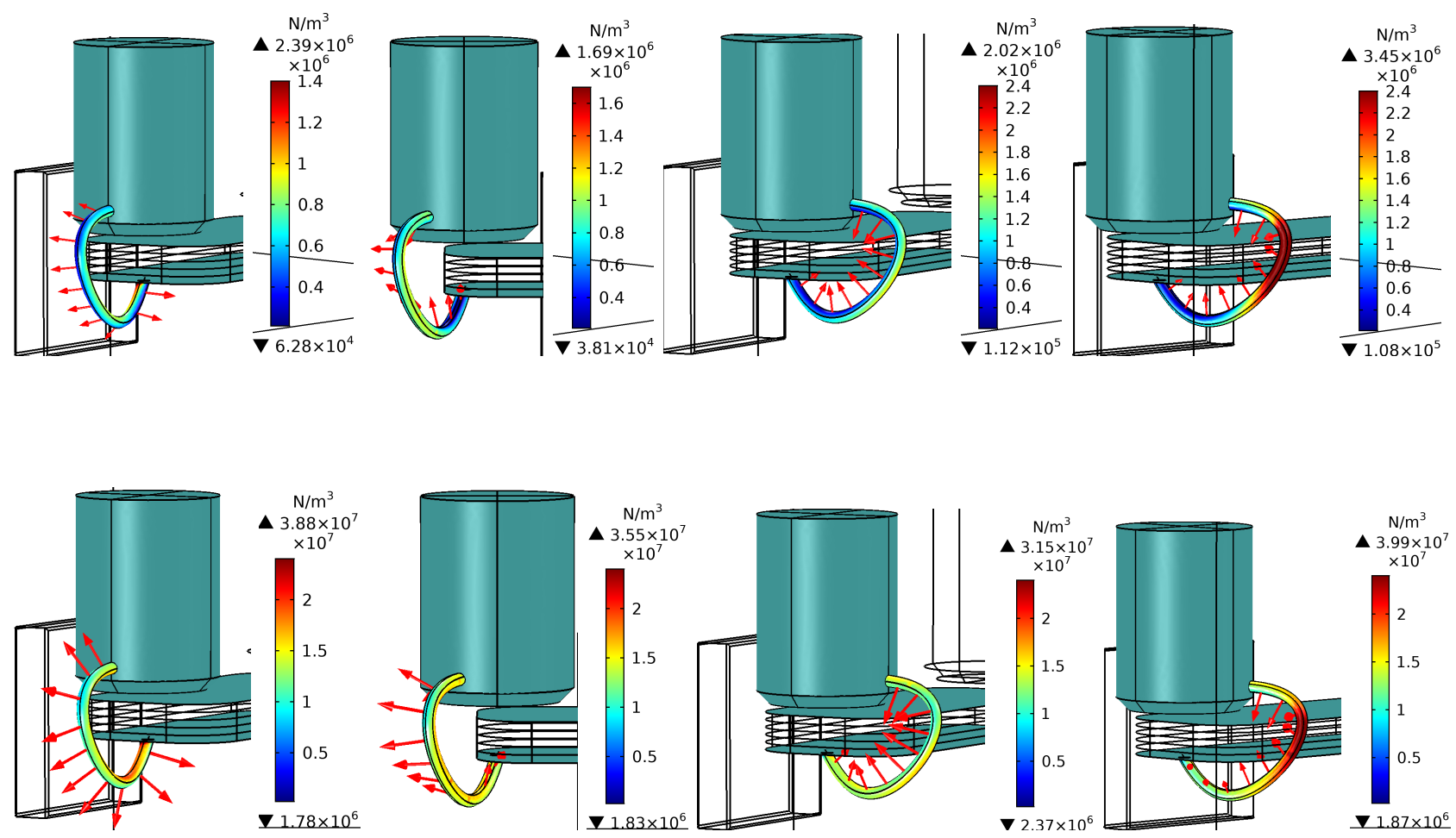

Figure 6. Lorentz force distribution in case with $80 \mathrm{~A}$ (upper row) and $400 \mathrm{~A}$ (lower row) current. Arc positions (from left to right) 0, 60, 90 and 120 degree. 